Tôhoku Math. Journ.

28 (1976), 563-581.

\title{
ON THE ABSOLUTE NÖRLUND SUMMABILITY FACTORS OF THE CONJUGATE SERIES OF A FOURIER SERIES
}

\section{YASUO OKUYAMA}

(Received June 7, 1975)

1. Let $\left\{s_{n}\right\}$ denote the $n$-th partial sum of a given infinite series $\sum a_{n}$. Let $\left\{p_{n}\right\}$ be a sequence of constants, real or complex, and let

$$
P_{n}=p_{0}+p_{1}+\cdots+p_{n} ; \quad P_{-k}=p_{-k}=0 \text {, for } k \geqq 1 \text {. }
$$

The sequence $\left\{t_{n}\right\}$, given by

$$
t_{n}=\frac{1}{P_{n}} \sum_{k=0}^{n} p_{n-k} s_{k}=\frac{1}{P_{n}} \sum_{k=0}^{n} P_{n-k} a_{k}, \quad\left(P_{n} \neq 0\right),
$$

defines the Nörlund means of the sequence $\left\{s_{n}\right\}$ generated by the sequence $\left\{p_{n}\right\}$.

Then, the series $\sum a_{n}$ is said to be summable $\left|N, p_{n}\right|$, if the sequence $\left\{t_{n}\right\}$ is of bounded variation, that is, the series

$$
\sum_{n}\left|t_{n}-t_{n-1}\right|
$$

is convergent.

In the special cases in which $p_{n}=\Gamma(n+\alpha) / \Gamma(\alpha) \Gamma(n+1), \alpha>0$, and $p_{n}=1 /(n+1)$, summability $\left|N, p_{n}\right|$ are the same as the summability $|C, \alpha|$ and the absolute harmonic summability, respectively.

Let $f(t)$ be a periodic function with period $2 \pi$ and integrable $(L)$ over $(-\pi, \pi)$. We assume without any loss of generality that the Fourier series of $f(t)$ is given by

$$
\sum_{n=1}^{\infty}\left(a_{n} \cos n t+b_{n} \sin n t\right)=\sum_{n=1}^{\infty} A_{n}(t)
$$

and $\int_{-\pi}^{\pi} f(t) d t=0$

The series "conjugate" to (1.3) is

$$
\sum_{n=1}^{\infty}\left(b_{n} \cos n t-a_{n} \sin n t\right)=\sum_{n=1}^{\infty} B_{n}(t) .
$$

We write 


$$
\begin{aligned}
& \varphi_{x}(t)=\varphi(t)=\frac{1}{2}\{f(x+t)+f(x-t)\} ; \\
& \psi_{x}(t)=\psi(t)=\frac{1}{2}\{f(x+t)-f(x-t)\} ; \\
& \left\{p_{n}\right\} \in \mathscr{C l}: p_{n+1} / p_{n} \leqq p_{n+2} / p_{n+1} \leqq 1 \quad(n=0,1,2, \cdots) ; \\
& \Delta p_{n}=p_{n}-p_{n+1} ; \\
& \tau=[\pi / t],
\end{aligned}
$$

i.e., the greatest integer in $\pi / t$.

2. Dealing with the absolute Nörlund summability of Fourier series, M. Izumi and S. Izumi [6] proved the following theorem.

THEOREM A. Let $\left\{p_{n}\right\}$ be non-negative and non-increasing and $\lambda(t)$, $t>0$, be a positive non-decreasing function such that $\{\lambda(n) /(n+1)\}$ is non-increasing,

$$
\sum_{k=n}^{\infty} \frac{\lambda(k)}{(k+1) P_{k}}=O\left(\frac{\lambda(n)}{P_{n}}\right), \quad n=0,1,2, \cdots
$$

and

$$
\int_{0}^{\pi} \lambda(C / t)|d \varphi(t)|<\infty \text { for some constant } C>0 .
$$

Then the series

$$
\sum_{n=1}^{\infty} \lambda(n) A_{n}(t)
$$

is summable $\left|N, p_{n}\right|$ at $t=x$.

This theorem is an extension of theorems due to L. S. Bosanquet [1] and M. Mohanty $[11,12]$.

Generalizing the theorems of O. P. Varshney [17] and T. Singh [16], K. Kanno [8] proved the following theorem.

THEOREM B. Let $\left\{p_{n}\right\}$ be non-negative and non-increasing. Let $\lambda(t)$, $t>0$, be a positive, non-decreasing function satisfying the condition $\left\{\lambda(n) / P_{n}\right\}$ is non-increasing.

If the conditions

$$
\sum_{k=n}^{\infty} \frac{p_{k} \lambda(k)}{P_{k}^{2}}=O\left(\frac{\lambda(n)}{P_{n}}\right), \quad n=0,1,2, \cdots
$$

and

$$
\int_{0}^{\pi} \lambda(C / t)|d \varphi(t)|<\infty
$$


for some constant $C>0$ hold, then the series

$$
\sum_{n=0}^{\infty} \frac{(n+1) p_{n}}{P_{n}} \lambda(n) A_{n+1}(t)
$$

is summable $\left|N, p_{n}\right|$ at $t=x$.

Very recently the author [13] generalized these theorems in the following form.

THEOREM C. Let $\left\{p_{n}\right\}$ be non-negative and non-increasing. Suppose that $\lambda(t), t>0$, is a positive non-decreasing function and $\{\mu(n)\}$ is a positive bounded sequence such that $\{\lambda(n) \mu(n) /(n+1)\}$ is non-increasing,

$$
\sum_{k=n}^{\infty} \frac{\lambda(k) \mu(k)}{k P_{k}}=O\left(\frac{\lambda(n)}{P_{n}}\right), \quad n=1,2, \cdots
$$

and

$$
\int_{0}^{\pi} \lambda(C / t)|d \varphi(t)|<\infty \text { for a constant } C>0 .
$$

Then the series

$$
\sum_{n=0}^{\infty} \lambda(n) \mu(n) A_{n+1}(t)
$$

is summable $\left|N, p_{n}\right|$ at $t=x$.

On the other hand, concerning the absolute Nörlund summability factor of the conjugate series of a Fourier series, A. Kumar [9] proved the following two theorems.

THEOREM D. Let $\left\{p_{n}\right\}$ and $\left\{\Delta p_{n}\right\}$ are both non-negative and nonincreasing sequences. Let $\lambda(t), t>0$, be a positive non-decreasing function such that $\{\lambda(t) / t\}$ is non-increasing for $t>0$.

If the conditions

$$
\begin{gathered}
\sum_{k=n}^{\infty} \frac{\lambda(k)}{k P_{k}}=O\left(\frac{\lambda(n)}{P_{n}}\right), \quad n=1,2, \cdots, \\
\int_{0}^{\pi} \lambda(C / t)|d \psi(t)|<\infty
\end{gathered}
$$

and

$$
\int_{0}^{\pi} \frac{\lambda(C / t)|\psi(t)|}{t} d t<\infty
$$

hold for some constant $C>\pi$, then the series

$$
\sum_{n=1}^{\infty} \lambda(n) B_{n}(t)
$$


is summable $\left|N, p_{n}\right|$ at $t=x$.

This theorem is the conjugate analogue of Theorem $\mathrm{A}$.

THEOREM E. Let $\left\{p_{n}\right\} \in \mathscr{M}$. If the conditions

$$
\sum_{k=n}^{\infty} \frac{1}{k P_{k} \log (k+1)}=O\left(\frac{1}{P_{n}}\right), \quad n=1,2, \cdots,
$$

$$
\psi(t) \in B V(0, \pi)
$$

and

$$
\int_{0}^{\pi} \frac{|\psi(t)|}{t \log (C / t)} d t<\infty
$$

for some constant $C>\pi$ hold, then the series

$$
\sum_{n=1}^{\infty} \frac{B_{n}(t)}{\log (n+1)}
$$

is summable $\left|N, p_{n}\right|$ at $t=x$.

Theorem $\mathrm{E}$ includes as special case the theorem of R. D. Ram and N. Lal [15], which is the conjugate analogue of the result due to O. P. Varschney [17].

Thus, comparing Theorem $\mathrm{C}$ with these theorems, we may expect a result for the $\left|N, p_{n}\right|$ summability of the series $\sum_{n=1}^{\infty} \lambda(n) \mu(n) B_{n}(x)$.

Our theorems are as follows:

THEOREM 1. Let $\left\{p_{n}\right\}$ and $\left\{\Delta p_{n}\right\}$ are both non-negative and nonincreasing. Assume that $\lambda(t), t>0$, is a positive non-decreasing function and $\mu(t), t>0$, is a positive bounded function such that $\{n \mu(n)\}$ and $\left\{\mu(n) / p_{n}\right\}$ are non-decreasing and $\{\lambda(t) \mu(t) / t\}$ is non-increasing.

If the conditions

$$
\begin{gathered}
\sum_{k=n}^{\infty} \frac{\lambda(k) \mu(k)}{k P_{k}}=O\left(\frac{\lambda(n)}{P_{n}}\right), \quad n=1,2, \cdots, \\
\int_{0}^{\pi} \frac{\lambda(C / t) \mu(C / t)|\psi(t)|}{t} d t<\infty
\end{gathered}
$$

and

$$
\int_{0}^{\pi} \lambda(C / t)|d \psi(t)|<\infty
$$

for some constant $C>\pi$ hold, then the series

$$
\sum_{n=1}^{\infty} \lambda(n) \mu(n) B_{n}(t)
$$


is summable $\left|N, p_{n}\right|$ at $t=x$.

If $\mu(t)=1$, our theorem reduces to Theorem D. If we put $\lambda(t)=1$ and $\mu(t)=1 / \log (t+1)$, then we see that our theorem partially includes Theorem E, because the condition $\left\{p_{n}\right\} \in \mathscr{M}$ implies that $\left\{\Delta p_{n}\right\}$ is nonnegative and non-increasing (see K. Kanno [8]).

THEOREM 2. Let $\left\{p_{n}\right\}$ be non-negative and non-increasing. Assume that $\lambda(t), t>0$, is a positive non-decreasing function and $\mu(n)$ is a positive bounded sequence such that $\{\lambda(n) \mu(n) / n\}$ is non-increasing.

If the conditions (2.4), (2.11) and

$$
\psi(+0)=0
$$

hold, then the series

$$
\sum_{n=1}^{\infty} \lambda(n) \mu(n) B_{n}(t)
$$

is summable $\left|N, p_{n}\right|$ at $t=x$.

Theorem 2 is a generalization of the theorem of H. P. Dikshit [5], which was given as the conjugate analogue of Theorem $B$.

3. We need some lemmas for the proofs of our theorems.

LEMMA 1 [4]. Let $\left\{p_{n}\right\}$ be a given sequence, then for any $x$, we have

$$
(1-x) \sum_{k=m}^{n} p_{k} x^{k}=p_{m} x^{m}-p_{n} x^{n+1}-\sum_{k=m}^{n-1} \Delta p_{k} x^{k+1}
$$

where $m$ and $n$ are integers such that $n \geqq m \geqq 0$.

LEMMA 2 [10]. If $\left\{p_{n}\right\}$ is non-negative and non-increasing, then for $0 \leqq a \leqq b<\infty, 0 \leqq t \leqq \pi$, and for any $n$, we have

$$
\left|\sum_{k=a}^{b} p_{k} \exp (i(n-k) t)\right| \leqq A P_{[1 / t]},
$$

where $A$ is a positive constant, not necessarily the same at each occurences.

LEMMA 3 [16]. If $\left\{p_{n}\right\}$ is non-negative and non-increasing, then $\left\{\left(P_{n}-P_{n-k}\right) / k\right\}$ is a non-decreasing sequence in $k$ for $1 \leqq k \leqq n$.

LEMMA 4 [16]. If $\left\{p_{n}\right\}$ and $\left\{\Delta p_{n}\right\}$ are both non-negative and nonincreasing, then for $1 \leqq k \leqq n$ and $n \geqq 2,\left\{\left(p_{n-k}-p_{n}\right) / k\right\}$ is nondecreasing.

LEMMA 5 [7]. If $\left\{p_{n}\right\}$ is non-negative and non-increasing, then $\left\{P_{n-k} / P_{n}\right\}$ is non-decreasing and $\left\{P_{n-k} / P_{n}\right\} \rightarrow 1$ as $n \rightarrow \infty$ for each fixed 
$k \geqq 0$.

LEMMA 6 (cf. [9], [18]). Let $\mu(t), t>0$, be a positive bounded function and $\lambda(t), t>0$, be a positive non-decreasing function such that $\{\lambda(t) \mu(t) / t\}$ is non-increasing and $\{n \mu(n)\}$ is non-decreasing.

If $\psi(t) \in B V(0, \pi)$ and

$$
\int_{0}^{\pi} \frac{\lambda(C / t) \mu(C / t)|\psi(t)|}{t} d t<\infty \text { for some constant } C>\pi \text { hold, }
$$

then the series

$$
\sum_{n=2}^{\infty} \frac{\lambda(n) \mu(n)\left|\psi\left(\theta_{n}\right)\right|}{n}
$$

converges where $\pi /(n+1) \leqq \theta_{n}<\pi / n, n=2,3, \cdots$.

Proof. Since the hypothesis $\psi(t) \in B V(0, \pi)$, implies that $|\psi(t)| \epsilon$ $B V(0, \pi)$, we can write

$$
|\psi(t)|=\psi_{1}(t)-\psi_{2}(t)
$$

where $\psi_{1}(t)$ and $\psi_{2}(t)$ are positive, bounded and non-decreasing functions. Using the condition that $\{\lambda(t) \mu(t) / t\}$ is positive and non-increasing, we have

$$
\begin{aligned}
\int_{\pi / n}^{\pi /(n-1)} & \frac{\psi_{1}(t)}{t^{2}}\left\{\frac{\lambda(C / t) \mu(C / t)}{1 / t}\right\} d t \\
& \geqq C \frac{\lambda\left(\frac{C}{\pi} n\right) \mu\left(\frac{C}{\pi} n\right)}{\frac{C}{\pi} n} \int_{\pi / n}^{\pi /(n-1)} \frac{\psi_{1}(t)}{t^{2}} d t \geqq \frac{\lambda\left(\frac{C}{\pi} n\right) \mu\left(\frac{C}{\pi} n\right)}{n} \psi_{1}\left(\frac{\pi}{n}\right)
\end{aligned}
$$

by virture of the fact that $\psi_{1}(t)$ is positive and non-decreasing.

Similarly, we have

$$
\begin{aligned}
\int_{\pi / n}^{\pi /(n-1)} \frac{\psi_{2}(t)}{t^{2}}\left\{\frac{\lambda(C / t) \mu(C / t)}{1 / t}\right\} d t & \\
\leqq & \frac{\lambda\left(\frac{C}{\pi}(n-1)\right) \mu\left(\frac{C}{\pi}(n-1)\right)}{\frac{C}{\pi}(n-1)} \int_{\pi / n}^{\pi /(n-1)} \frac{\psi_{2}(t)}{t^{2}} d t \\
& \leqq \frac{\lambda\left(\frac{C}{\pi}(n-1)\right) \mu\left(\frac{C}{\pi}(n-1)\right)}{(n-1)} \psi_{2}\left(\frac{\pi}{n-1}\right) .
\end{aligned}
$$

If we put $\alpha=C / \pi$, then we obtain by (3.1) and (3.2) 


$$
\begin{aligned}
& \frac{\lambda(\alpha n) \mu(\alpha n)}{n} \psi_{1}\left(\frac{\pi}{n}\right)-\frac{\lambda(\alpha(n-1)) \mu(\alpha(n-1))}{(n-1)} \psi_{2}\left(\frac{\pi}{n-1}\right) \\
& \leqq \int_{\pi / n}^{\pi /(n-1)} \frac{\left\{\psi_{1}(t)-\psi_{2}(t)\right\}}{t} \lambda(C / t) \mu(C / t) d t
\end{aligned}
$$

Since $\pi /(n+1) \leqq \theta_{n}<\pi / n$, we have

(3.4) $\frac{\lambda(\alpha n) \mu(\alpha n)}{n}\left|\psi\left(\theta_{n}\right)\right|$

$$
\begin{aligned}
= & \frac{\lambda(\alpha n) \mu(\alpha n)}{n}\left\{\psi_{1}\left(\theta_{n}\right)-\psi_{2}\left(\theta_{n}\right)\right\} \\
\leqq & \frac{\lambda(\alpha n) \mu(\alpha n)}{n}\left\{\psi_{1}\left(\frac{\pi}{n}\right)-\psi_{2}\left(\frac{\pi}{n+1}\right)\right\} \\
= & \frac{\lambda(\alpha n) \mu(\alpha n)}{n} \psi_{1}\left(\frac{\pi}{n}\right)-\frac{\lambda(\alpha(n-1)) \mu(\alpha(n-1))}{(n-1)} \psi_{2}\left(\frac{\pi}{n-1}\right) \\
& +\frac{\lambda(\alpha(n-1)) \mu(\alpha(n-1))}{(n-1)} \psi_{2}\left(\frac{\pi}{n-1}\right)-\frac{\lambda(\alpha(n-1)) \mu(\alpha(n-1))}{(n-1)} \psi_{2}\left(\frac{\pi}{n}\right) \\
& +\frac{\lambda(\alpha(n-1)) \mu(\alpha(n-1))}{(n-1)} \psi_{2}\left(\frac{\pi}{n}\right)-\frac{\lambda(\alpha n) \mu(\alpha n)}{n} \psi_{2}\left(\frac{\pi}{n+1}\right) .
\end{aligned}
$$

Thus, since $\{n \mu(n)\}$ is non-decreasing, we have by (3.3) and (3.4)

$$
\begin{aligned}
\sum_{n=2}^{\infty} & \frac{\lambda(n) \mu(n)\left|\psi\left(\theta_{n}\right)\right|}{n} \\
\leqq & A \sum_{n=2}^{\infty} \frac{\lambda(\alpha n) \mu(\alpha n)\left|\psi\left(\theta_{n}\right)\right|}{n} \\
\leqq & A \sum_{n=2}^{\infty}\left\{\frac{\lambda(\alpha n) \mu(\alpha n)}{n} \psi_{1}\left(\frac{\pi}{n}\right)-\frac{\lambda(\alpha(n-1)) \mu(\alpha(n-1))}{(n-1)} \psi_{2}\left(\frac{\pi}{n-1}\right)\right\} \\
& +A \sum_{n=2}^{\infty} \frac{\lambda(\alpha(n-1)) \mu(\alpha(n-1))}{(n-1)} \Delta\left\{\psi_{2}\left(\frac{\pi}{n-1}\right)\right\} \\
& \left.+A \sum_{n=2}^{\infty} \Delta \frac{\lambda(\alpha(n-1)) \mu(\alpha(n-1))}{\alpha(n-1)} \psi_{2}\left(\frac{\pi}{n}\right)\right\} \\
\leqq & A \sum_{n=2}^{\infty} \int_{\pi / n}^{\pi /(n-1)} \frac{\left\{\psi_{1}(t)-\psi_{2}(t)\right\}}{t} \lambda(C / t) \mu(C / t) d t \\
& +A \lambda(\alpha) \mu(\alpha) \sum_{n=2}^{\infty} \Delta\left\{\psi_{2}\left(\frac{\pi}{n-1}\right)\right\} \\
& +A \frac{\lambda(\alpha) \mu(\alpha)}{\alpha} \psi_{2}\left(\frac{\pi}{2}\right) \\
\leqq & A \int_{0}^{\pi} \frac{\lambda(C / t) \mu(C / t)|\psi(t)|}{t} d t+A<\infty
\end{aligned}
$$


by virture of the hypotheses (2.10) and that $\{\lambda(n) \mu(n) / n\}$ is non-increasing and $\psi_{2}(t)$ is non-decreasing and bounded.

LEMma 7. Let $\left\{p_{n}\right\}$ be a sequence of positive numbers. Then the condition

$$
\sum_{k=n}^{\infty} \frac{\mu(k)}{k P_{k}}=O\left(\frac{1}{P_{n}}\right)
$$

is equivalent to the condition

$$
\sum_{k=1}^{n} \frac{\mu(k) P_{k}}{k}=O\left(P_{n}\right)
$$

where $\{\mu(n)\}$ is a positive sequence.

Proof. First, we assume that the condition (3.5) holds.

Using the formula

$$
\sum_{k=m+1}^{n} a_{k} b_{k}=\sum_{k=m+2}^{n}\left(a_{k}-a_{k-1}\right) \sum_{r=k}^{\infty} b_{r}+a_{m+1} \sum_{r=m+1}^{\infty} b_{r}-a_{n} \sum_{r=n+1}^{\infty} b_{r},
$$

we have

$$
\begin{aligned}
& \frac{1}{P_{n}} \sum_{k=1}^{n} \frac{\mu(k) P_{k}}{k}=\frac{1}{P_{n}} \sum_{k=1}^{n} P_{k}^{2} \frac{\mu(k)}{k P_{k}} \\
& \quad=\frac{1}{P_{n}} \sum_{k=2}^{n}\left(P_{k}^{2}-P_{k-1}^{2}\right) \sum_{r=k}^{\infty} \frac{\mu(r)}{r P_{r}}+\frac{P_{1}^{2}}{P_{n}} \sum_{r=1}^{\infty} \frac{\mu(r)}{r P_{r}}-P_{n} \sum_{r=n+1}^{\infty} \frac{\mu(r)}{r P_{r}} \\
& \quad=W_{1}+W_{2}-W_{3},
\end{aligned}
$$

say. Clearly, we have

$$
W_{2}=O(1) \text { and } W_{3}=O(1) .
$$

Also, we have

$$
\begin{aligned}
W_{1} & \leqq \frac{1}{P_{n}} \sum_{k=2}^{n} p_{k}\left(P_{k}+P_{k-1}\right) \sum_{r=k}^{\infty} \frac{\mu(r)}{r P_{r}} \\
& \leqq A \frac{1}{P_{n}} \sum_{k=2}^{n} p_{k} \\
& =O(1) .
\end{aligned}
$$

Collecting the above estimations, we have the condition (3.6).

The inverse statement is proved similarly.

Thus, we have by Lemma 7

LeMma 8. The condition (2.4) implies the condition (3.6).

4. Proof of Theorem 1. By (1.1) we have

$$
\tilde{t}_{n}=\frac{1}{P_{n}} \sum_{k=0}^{n} P_{n-k} \lambda(k) \mu(k) B_{k}(x)
$$


where

$$
B_{k}(x)=\frac{2}{\pi} \int_{0}^{\pi} \psi(t) \sin k t d t
$$

Hence

$$
\begin{aligned}
\tilde{t}_{n} & -\tilde{t}_{n-1} \\
& =\frac{2}{\pi} \int_{0}^{\pi} \psi(t)\left\{\frac{1}{P_{n} P_{n-1}} \sum_{k=1}^{n}\left(P_{n} p_{n-k}-P_{n-k} p_{n}\right) \lambda(k) \mu(k) \sin k t\right\} d t .
\end{aligned}
$$

Therefore, to prove the theorem, we have to show that

$$
\begin{aligned}
I & =\sum_{n=1}^{\infty}\left|\tilde{t}_{n}-\tilde{t}_{n-1}\right| \\
& =\sum_{n=1}^{\infty}\left|\int_{0}^{\pi} \psi(t)\left\{\frac{1}{P_{n} P_{n-1}} \sum_{k=1}^{n}\left(P_{n} p_{n-k}-P_{n-k} p_{n}\right) \lambda(k) \mu(k) \sin k t\right\} d t\right| \\
& =O(1) .
\end{aligned}
$$

Now taking $\theta_{n}$ as points of continuity of $\psi(t)$ with $\pi /(n+1) \leqq$ $\theta_{n}<\pi / n$ for $n=1,2, \cdots$, we obtain

$$
\begin{aligned}
I \leqq & \sum_{n=1}^{\infty}\left|\int_{0}^{\theta_{n}} \psi(t)\left\{\frac{1}{P_{n} P_{n-1}} \sum_{k=1}^{n}\left(P_{n} p_{n-k}-P_{n-k} p_{n}\right) \lambda(k) \mu(k) \sin k t\right\} d t\right| \\
& +\sum_{n=1}^{\infty}\left|\int_{\theta_{n}}^{\pi} \psi(t)\left\{\frac{1}{P_{n} P_{n-1}} \sum_{k=1}^{n}\left(P_{n} p_{n-k}-P_{n-k} p_{n}\right) \lambda(k) \mu(k) \sin k t\right\} d t\right| \\
= & I_{1}+I_{2},
\end{aligned}
$$

say. We define $H_{n}(t)$ in the following way;

$$
H_{n}(t)=\left\{\begin{array}{l}
\frac{1}{P_{n} P_{n-1}} \sum_{k=1}^{n}\left(P_{n} p_{n-k}-P_{n-k} p_{n}\right) \lambda(k) \mu(k) \sin k t \text { for } 0 \leqq t<\theta_{n} \\
0 \text { for } \theta_{n} \leqq t \leqq \pi .
\end{array}\right.
$$

Since $\theta_{n}<\pi / n$ and $H_{n}(t)=0$ for $t \geqq \theta_{n}$, then we have

$$
H_{n}(t)=0 \text { for } n \geqq \tau+1 \text {. }
$$

Therefore, since $\{\lambda(k)\}$ is non-decreasing, $\{k \mu(k)\}$ is non-decreasing and $|\sin k t| \leqq k t$, we have

$$
\begin{aligned}
\sum_{n=1}^{\infty}\left|H_{n}(t)\right| & =\sum_{n=1}^{\tau}\left|H_{n}(t)\right| \\
& \leqq \sum_{n=1}^{\tau} \sum_{k=1}^{n}\left(\frac{P_{n-k}}{P_{n}}-\frac{P_{n-k-1}}{P_{n-1}}\right) \lambda(k) \mu(k)|\sin k t| \\
& =\sum_{k=1}^{\tau} \lambda(k) \mu(k)|\sin k t| \sum_{n=k}^{\tau}\left(\frac{P_{n-k}}{P_{n}}-\frac{P_{n-k-1}}{P_{n-1}}\right)
\end{aligned}
$$




$$
\begin{aligned}
& \leqq t \sum_{k=1}^{\tau} k \lambda(k) \mu(k) \frac{P_{\tau-k}}{P_{\tau}} \\
& \leqq A \tau \lambda(C / t) \mu(C / t)
\end{aligned}
$$

by virture of Lemma 5 .

By (4.2) and (2.10), we have

$$
\begin{aligned}
I_{1} & =\sum_{n=1}^{\infty}\left|\int_{0}^{\pi} \psi(t) H_{n}(t) d t\right| \leqq \int_{0}^{\pi}|\psi(t)| \sum_{n=1}^{\infty}\left|H_{n}(t)\right| d t \\
& \leqq A \int_{0}^{\pi} \frac{\psi(t) \mid \lambda(C / t) \mu(C / t)}{t} d t<\infty .
\end{aligned}
$$

Now, observing that $\psi(\pi)=0$ and integrating by parts, we have

$$
\begin{aligned}
I_{2} \leqq & \sum_{n=1}^{\infty}\left|\psi\left(\theta_{n}\right)\right|\left|\frac{1}{P_{n} P_{n-1}} \sum_{k=1}^{n}\left(P_{n} p_{n-k}-p_{n} P_{n-k}\right) \frac{\lambda(k) \mu(k) \cos \left(k \theta_{n}\right)}{k}\right| \\
& +\sum_{n=1}^{\infty}\left|\int_{\theta_{n}}^{\pi} \sum_{k=1}^{n}\left(\frac{P_{n-k^{\prime}}}{P_{n}}-\frac{P_{n-k-1}}{P_{n-1}}\right) \frac{\lambda(k) \mu(k) \cos k t}{k} d \psi(t)\right| \\
= & I_{21}+I_{22},
\end{aligned}
$$

say. Let us write $m=[n / 2]$. Then we obtain

$$
\begin{aligned}
I_{21} \leqq & \sum_{n=2}^{\infty} \frac{\left|\psi\left(\theta_{n}\right)\right|}{P_{n} P_{n-1}}\left|\sum_{k=1}^{m} \frac{P_{n}-P_{n-k}}{k} p_{n-k} \lambda(k) \mu(k) \cos \left(k \theta_{n}\right)\right| \\
& +\sum_{n=2}^{\infty} \frac{\left|\psi\left(\theta_{n}\right)\right|}{P_{n} P_{n-1}}\left|\sum_{k=1}^{m} \frac{p_{n-k}-p_{n}}{k} P_{n-k} \lambda(k) \mu(k) \cos \left(k \theta_{n}\right)\right| \\
& +\sum_{n=2}^{\infty}\left|\psi\left(\theta_{n}\right)\right|\left|\sum_{k=m+1}^{n} \frac{P_{n} p_{n-k}-P_{n-k} p_{n}}{P_{n} P_{n-1}} \frac{\lambda(k) \mu(k)}{k} \cos \left(k \theta_{n}\right)\right|+A \\
= & I_{211}+I_{212}+I_{213}+A,
\end{aligned}
$$

say. Since $\left\{\mu(n) / p_{n}\right\}$ is non-decreasing and $\{n \lambda(n) \mu(n)\}$ is non-decreasing, we have by Lemma 3

$$
\begin{aligned}
I_{211} & \leqq \sum_{n=2}^{\infty} \frac{\left|\psi\left(\theta_{n}\right)\right|}{P_{n} P_{n-1}} \sum_{k=1}^{m} \frac{P_{n}-P_{n-k}}{k} p_{n-k} \lambda(k) \frac{\mu(k)}{p_{k}} p_{k} \\
& \leqq \sum_{n=2}^{\infty} \frac{\left|\psi\left(\theta_{n}\right)\right|}{P_{n} P_{n-1}} \frac{P_{n}-P_{n-m}}{m} \lambda(m) \frac{\mu(m)}{p_{m}} p_{n-m} \sum_{k=1}^{m} p_{k} \\
& \leqq \sum_{n=2}^{\infty} \frac{\lambda(m) \mu(m)\left|\psi\left(\theta_{n}\right)\right|}{m} \\
& \leqq A \sum_{n=2}^{\infty} \frac{\lambda(n) \mu(n)\left|\psi\left(\theta_{n}\right)\right|}{n}<\infty
\end{aligned}
$$

by virture of Lemma 6 .

Similarly, by Lemma 4, we have 


$$
\begin{aligned}
I_{212} & \leqq \sum_{n=2}^{\infty} \frac{\left|\psi\left(\theta_{n}\right)\right|}{P_{n} P_{n-1}} \sum_{k=1}^{m} \frac{p_{n-k}-p_{n}}{k} P_{n-k} \lambda(k) \frac{\mu(k)}{p_{k}} p_{k} \\
& \leqq \sum_{n=2}^{\infty} \frac{\left|\psi\left(\theta_{n}\right)\right|}{P_{n} P_{n-1}} \frac{p_{n-m}-p_{n}}{m} \lambda(m) \frac{\mu(m)}{p_{m}} P_{n-1} \sum_{k=1}^{m} p_{k} \\
& \leqq \sum_{n=2}^{\infty} \frac{\lambda(m) \mu(m)\left|\psi\left(\theta_{n}\right)\right|}{m} \\
& \leqq A \sum_{n=2}^{\infty} \frac{\lambda(n) \mu(n)\left|\psi\left(\theta_{n}\right)\right|}{n}<\infty
\end{aligned}
$$

by virture of Lemma 6 .

Since $\{\lambda(n) \mu(n) / n\}$ is non-increasing and $\{n \lambda(n) \mu(n)\}$ is non-decreasing, we have

$$
\begin{aligned}
I_{213} & \leqq \sum_{n=2}^{\infty}\left|\psi\left(\theta_{n}\right)\right| \sum_{k=m+1}^{n} \frac{P_{n} p_{n-k}-P_{n-k} p_{n}}{P_{n} P_{n-1}} \frac{\lambda(k) \mu(k)}{k} \\
& \leqq \sum_{n=2}^{\infty} \frac{\left|\psi\left(\theta_{n}\right)\right| \lambda(m) \mu(m)}{m} \sum_{k=m+1}^{n} \frac{P_{n} p_{n-k}-P_{n-k} p_{n}}{P_{n} P_{n-1}} \\
& \leqq \sum_{n=2}^{\infty} \frac{\left|\psi\left(\theta_{n}\right)\right| \lambda(m) \mu(m)}{m P_{n-1}} \sum_{k=m+1}^{n} p_{n-k} \\
& \leqq \sum_{n=2}^{\infty} \frac{\lambda(m) \mu(m)\left|\psi\left(\theta_{n}\right)\right|}{m} \\
& \leqq A \sum_{n=2}^{\infty} \frac{\lambda(n) \mu(n)\left|\psi\left(\theta_{n}\right)\right|}{n}<\infty
\end{aligned}
$$

by virture of Lemma 6 . Thus, by $I_{211}, I_{212}$ and $I_{213}$, we prove the finiteness of $I_{21}$.

Next, we define $K_{n}(t)$ in the following way;

$$
K_{n}(t)=\left\{\begin{array}{l}
0 \quad \text { for } 0 \leqq t<\theta_{n} \\
\sum_{k=1}^{n}\left(\frac{P_{n-k}}{P_{n}}-\frac{P_{n-k-1}}{P_{n-1}}\right) \frac{\lambda(k) \mu(k) \cos k t}{k} \text { for } \theta_{n} \leqq t \leqq \pi
\end{array}\right.
$$

Thus, we have

$$
\begin{aligned}
I_{22} & \leqq \sum_{n=1}^{\infty} \int_{0}^{\pi}\left|K_{n}(t)\right||d \psi(t)| \\
& =\int_{0}^{\pi} \sum_{n=1}^{\infty}\left|K_{n}(t)\right||d \psi(t)| .
\end{aligned}
$$

Considering the condition (2.11) and the definition of $K_{n}(t)$, it suffices for $I_{22}<\infty$ to prove that uniformly in $0<t \leqq \pi$, 


$$
\begin{aligned}
J & =\sum_{n=\tau}^{\infty}\left|\sum_{k=1}^{n}\left(\frac{P_{n-k}}{P_{n}}-\frac{P_{n-k-1}}{P_{n-1}}\right) \lambda(k) \mu(k) \frac{\cos k t}{k}\right| \\
& =O(\lambda(C / t)) .
\end{aligned}
$$

Now, we have

$$
\begin{aligned}
J \leqq & \sum_{n=\tau}^{2 \tau}\left|\sum_{k=1}^{n}\left(\frac{P_{n-k}}{P_{n}}-\frac{P_{n-k-1}}{P_{n-1}}\right) \frac{\lambda(k) \mu(k)}{k} \cos k t\right| \\
& +\sum_{n=2 \tau+1}^{\infty}\left|\sum_{k=1}^{\tau}\left(\frac{P_{n-k}}{P_{n}}-\frac{P_{n-k-1}}{P_{n-1}}\right) \frac{\lambda(k) \mu(k)}{k} \cos k t\right| \\
& +\sum_{n=2 \tau+1}^{\infty}\left|\sum_{k=\tau+1}^{n}\left(\frac{P_{n-k}}{P_{n}}-\frac{P_{n-k-1}}{P_{n-1}}\right) \frac{\lambda(k) \mu(k)}{k} \cos k t\right| \\
= & J_{1}+J_{2}+J_{3},
\end{aligned}
$$

say. Thus we have

$$
\begin{aligned}
J_{1} \leqq & \sum_{n=\tau}^{2 \tau} \sum_{k=1}^{n}\left(\frac{P_{n-k}}{P_{n}}-\frac{P_{n-k-1}}{P_{n-1}}\right) \frac{\lambda(k) \mu(k)}{k} \\
\leqq & \sum_{k=1}^{\tau} \frac{\lambda(k) \mu(k)}{k} \sum_{n=\tau}^{2 \tau}\left(\frac{P_{n-k}}{P_{n}}-\frac{P_{n-k-1}}{P_{n-1}}\right) \\
& +\sum_{k=\tau}^{2 \tau} \frac{\lambda(k) \mu(k)}{k} \sum_{n=k}^{2 \tau}\left(\frac{P_{n-k}}{P_{n}}-\frac{P_{n-k-1}}{P_{n-1}}\right) \\
= & J_{11}+J_{12},
\end{aligned}
$$

say. Since $1-P_{\tau-k-1} / P_{\tau-1} \leqq P_{k} / P_{\tau-1}$, we have by Lemmas 5 and 8

$$
\begin{aligned}
J_{11} & \leqq \sum_{k=1}^{\tau} \frac{\lambda(k) \mu(k)}{k}\left(1-\frac{P_{\tau-k-1}}{P_{\tau-1}}\right) \\
& \leqq \frac{\lambda(\tau)}{P_{\tau-1}} \sum_{k=1}^{\tau} \frac{\mu(k) P_{k}}{k} \\
& =O(\lambda(C / t))
\end{aligned}
$$

by virture of the hypothesis that $\{\lambda(n)\}$ is non-decreasing.

Also, since $\{\lambda(n) \mu(n) / n\}$ is non-increasing and $\{\mu(n)\}$ is bounded, we have

$$
\begin{aligned}
J_{12} & \leqq \sum_{k=\tau}^{2 \tau} \frac{\lambda(k) \mu(k)}{k} \frac{P_{2 \tau-k}}{P_{2 \tau}} \\
& \leqq A \frac{\lambda(\tau) \mu(\tau)}{\tau} \sum_{k=\tau}^{2 \tau} 1 \\
& =O(\lambda(C / t)) .
\end{aligned}
$$

Hence we have by $J_{11}$ and $J_{12}$

$$
J_{1}=O(\lambda(C / t))
$$


By the same method as $J_{11}$, we have

$$
\begin{aligned}
J_{2} & \leqq \sum_{k=1}^{\tau} \frac{\lambda(k) \mu(k)}{k} \sum_{n=2 \tau+1}^{\infty}\left(\frac{P_{n-k}}{P_{n}}-\frac{P_{n-k-1}}{P_{n-1}}\right) \\
& \leqq \sum_{k=1}^{\tau} \frac{\lambda(k) \mu(k)}{k}\left(1-\frac{P_{2 \tau-k}}{P_{2 \tau}}\right) \\
& \leqq \frac{\lambda(\tau)}{P_{2 \tau}} \sum_{k=1}^{\tau} \frac{\mu(k) P_{k}}{k} \\
& \leqq A \frac{\lambda(\tau)}{P_{2 \tau}} P_{\tau} \\
& =O(\lambda(C / t)) .
\end{aligned}
$$

In order to show that $J_{3}=O(\lambda(C / t))$, we consider the sum

$$
J_{3}^{*}=\sum_{n=2 \tau+1}^{N}\left|\sum_{k=\tau+1}^{n}\left(\frac{P_{n-k}}{P_{n}}-\frac{P_{n-k-1}}{P_{n-1}}\right) \lambda(k) \mu(k) \frac{\exp (i k t)}{k}\right| \text {. }
$$

Then it is enough to prove that

$$
J_{3}^{*}=O(\lambda(C / t)) \text { as } N \rightarrow \infty \text {. }
$$

This is similarly proved by the same method as that used by $\mathrm{Y}$. Okuyama [13].

Collecting the estimations $J_{1}, J_{2}$ and $J_{3}$, we have

$$
J=O(\lambda(C / t)) \text {. }
$$

Thus, by the hypothesis (2.11), we see that $I_{22}$ is finite.

Therefore, by the above estimations, our theorem is completely proved.

5. Proof of Theorem 2. We shall only sketch the proof. By (4.1), we have

$$
\widetilde{I}=\sum_{n=1}^{\infty}\left|\tilde{t}_{n}-\tilde{t}_{n-1}\right|=\sum_{n=1}^{\infty} \frac{1}{P_{n} P_{n-1}}\left|C_{n}\right|
$$

where

$$
C_{n}=\frac{2}{\pi} \int_{0}^{\pi} \psi(t)\left\{\sum_{k=1}^{n}\left(P_{n} p_{n-k}-P_{n-k} p_{n}\right) \lambda(k) \mu(k) \sin k t\right\} d t
$$

Since $\psi(\pi)=\psi(0)=\psi(+0)$, we have $\int_{0}^{\pi} d \psi(t)=0$, and therefore by integration by parts, we have (cf. T. Pati [14])

$$
\left|C_{n}\right| \leqq \frac{2}{\pi} \int_{0}^{\pi}\left|d_{n}(t)\right||d \psi(t)|
$$


for each $n$, where

$$
\left|d_{n}(t)\right|= \begin{cases}\text { either } & \left|\sum_{k=1}^{n}\left(P_{n} p_{n-k}-p_{n} P_{n-k}\right) \lambda(k) \mu(k) \frac{1-\cos k t}{k}\right| \\ \text { or } & \left|\sum_{k=1}^{n}\left(P_{n} p_{n-k}-p_{n} P_{n-k}\right) \lambda(k) \mu(k) \frac{\cos k t}{k}\right| .\end{cases}
$$

Thus we have

$$
\begin{aligned}
\widetilde{I} \leqq & \frac{2}{\pi} \int_{0}^{\pi} \sum_{n=1}^{\tau-1} \frac{1}{P_{n} P_{n-1}}\left|\sum_{k=1}^{n}\left(P_{n} p_{n-k}-p_{n} P_{n-k}\right) \lambda(k) \mu(k) \frac{1-\cos k t}{k}\right||d \psi(t)| \\
& +\frac{2}{\pi} \int_{0}^{\pi} \sum_{n=\tau}^{\infty}\left|\sum_{k=1}^{n}\left(\frac{P_{n-k}}{P_{n}}-\frac{P_{n-k-1}}{P_{n-1}}\right) \lambda(k) \mu(k) \frac{\cos k t}{k}\right||d \psi(t)| .
\end{aligned}
$$

Since by the hypothesis $\int_{0}^{\pi} \lambda(C / t)|d \psi(t)|<\infty$, in order to prove the finiteness of $\widetilde{I}$, it is enough to show that uniformly in $0<t \leqq \pi$

$$
\tilde{I}_{1}=\sum_{n=1}^{\tau-1} \frac{1}{P_{n} P_{n-1}}\left|\sum_{k=1}^{n}\left(P_{n} p_{n-k}-p_{n} P_{n-k}\right) \lambda(k) \mu(k) \frac{1-\cos k t}{k}\right|=O(\lambda(C / t))
$$

and

$$
\widetilde{I}_{2}=\sum_{n=\tau}^{\infty}\left|\sum_{k=1}^{n}\left(\frac{P_{n-k}}{P_{n}}-\frac{P_{n-k-1}}{P_{n-1}}\right) \lambda(k) \mu(k) \frac{\cos k t}{k}\right|=O(\lambda(C / t)) .
$$

Since $1-\cos k t \leqq k^{2} t^{2}$, we have

$$
\begin{aligned}
\widetilde{I}_{1} & \leqq A t^{2} \sum_{n=1}^{\tau-1} \frac{1}{P_{n-1}} \sum_{k=1}^{n} p_{n-k} \lambda(k) \mu(k) k \\
& \leqq A t^{2} \sum_{n=1}^{\tau-1} n \lambda(n) \\
& =O(\lambda(C / t))
\end{aligned}
$$

by the hypotheses that $\{\lambda(n)\}$ is non-decreasing and $\{\mu(n)\}$ is bounded.

$\widetilde{I}_{2}$ follows directly from the estimation $J$ of the proof of Theorem 1 . Hence we complete the proof of Theorem 2.

6. In this section, we shall establish the theorem which will clarify the relation between Theorem 1 and Theorem 2 .

THEOREM 3. Let $\lambda(t), t>0$, be a non-decreasing function tending to an infinity as $t \rightarrow \infty$ and $\mu(t), t>0$, be a positive function. If the condition

$$
\frac{d}{d t} \lambda(C / t)=\gamma \frac{\lambda(C / t) \mu(C / t)}{t}
$$

holds for a suitable constant $\gamma$, then the set of the conditions (2.10) and 
(2.11) is equivalent to the set of the conditions (2.11) and (2.12).

To prove this theorem, we require the following two lemmas.

LEMMA 9. Let $\lambda(t)$ be a non-decreasing function and $\mu(t)$ be a positive function. If the condition

$$
\frac{d}{d t} \lambda(C / t)=\gamma \frac{\lambda(C / t) \mu(C / t)}{t}
$$

holds for a suitable constant $\gamma$, then the condition

$$
\lambda(C / t) \psi(t) \in B V(0, \pi)
$$

is equivalent to the condition (2.11) wherever the condition (2.10) holds.

Proof. This lemma easily follows from the fact that

$$
\begin{aligned}
\int_{0}^{\pi} \lambda(C / t)|d \psi(t)| & \leqq \int_{0}^{\pi}|d\{\lambda(C / t) \psi(t)\}|+\int_{0}^{\pi}|\psi(t)||d \lambda(C / t)| \\
& =\int_{0}^{\pi}|d\{\lambda(C / t) \psi(t)\}|+|\gamma| \int_{0}^{\pi} \frac{\lambda(C / t) \mu(C / t)|\psi(t)|}{t} d t<\infty
\end{aligned}
$$

and

$$
\begin{aligned}
\int_{0}^{\pi}|d\{\lambda(C / t) \psi(t)\}| & \leqq \int_{0}^{\pi} \lambda(C / t)|d \psi(t)|+\int_{0}^{\pi}|\psi(t)||d \lambda(C / t)| \\
& \leqq \int_{0}^{\pi} \lambda(C / t)|d \psi(t)|+|\gamma| \int_{0}^{\pi} \frac{\lambda(C / t) \mu(C / t)|\psi(t)|}{t} d t<\infty .
\end{aligned}
$$

LEMmA 10. Under the same assumptions as those of Theorem 3, the set of the conditions (2.10) and (6.1) is equivalent to the set of the conditions (2.11) and (2.12).

Proof. If the conditions (2.11) and (2.12) hold, then we have

$$
\begin{aligned}
\int_{0}^{\pi} \frac{\lambda(C / t) \mu(C / t)|\psi(t)|}{t} d t & =\int_{0}^{\pi} \frac{\lambda(C / t) \mu(C / t)}{t} d t\left|\int_{0}^{t} d \psi(u)\right| \\
& \leqq \int_{0}^{\pi} \frac{\lambda(C / t) \mu(C / t)}{t} d t \int_{0}^{t}|d \psi(u)| \\
& =\int_{0}^{\pi}|d \psi(u)| \int_{u}^{\pi} \frac{\lambda(C / t) \mu(C / t)}{t} d t \\
& \leqq A \int_{0}^{\pi}|d \psi(u)|\left|\int_{u}^{\pi} d \lambda(C / t)\right| \\
& =A \int_{0}^{\pi}|d \psi(u)|(\lambda(C / u)-\lambda(C / \pi))<\infty
\end{aligned}
$$

from which (2.10) follows; and then (6.1) follows from (6.3). 
Conversely, suppose that the conditions (2.10) and (6.1) hold. Then we obtain (2.11) from (6.2). Since $\lambda(t)$ is a non-decreasing function tending to an infinity as $t \rightarrow \infty,(2.12)$ is obvious from (6.1).

Proof of Theorem 3. By Lemmas 9 and 10, we complete the proof of our theorem.

7. In this section, we consider some applications of our theorems and deduce the several corollaries from the theorems.

Our results are as follows:

CoRollary 1. If

$$
\int_{0}^{\pi} t^{-1-\alpha}|\psi(t)| d t<\infty \quad\left(\text { or } \quad\left(\mathrm{i}^{\prime}\right) \psi(+0)=0\right)
$$

and

$$
\int_{0}^{\pi} t^{-\alpha}|d \psi(t)|<\infty,
$$

then the series $\sum_{n=1}^{\infty} n^{\alpha} B_{n}(t)$ is summable $|C, \beta|$ at $t=x$, where

$$
0 \leqq \alpha<\beta<1 \text {. }
$$

This corollary coincides to L. S. Bosanquet and J. M. Hyslop [2] for $\alpha=0$. By Theorem 3, Corollary 1 coincides to R. Mohanty [11] for $0<\alpha<1$. Also, see H. P. Dikshit [3].

COROLLARY 2. If

$$
\int_{0}^{\pi} t^{-1}(\log C / t)^{\beta}|\psi(t)| d t<\infty \quad\left(\text { or } \quad\left(\mathrm{i}^{\prime}\right) \psi(+0)=0\right)
$$

and

$$
\int_{0}^{\pi}(\log C / t)^{\beta}|d \psi(t)|<\infty,
$$

then the series $\sum_{n=1}^{\infty}(\log n)^{\beta} B_{n}(t)$ is summable $|C, \alpha|$ at $t=x$, where $0<\alpha<1$ and $\beta \geqq 0$.

COROLLARY 3. If

$$
\int_{0}^{\pi} t^{-1}(\log C / t)^{\beta-1}|\psi(t)| d t<\infty \quad\left(\text { or } \quad\left(\mathrm{i}^{\prime}\right) \psi(+0)=0\right)
$$

and

$$
\int_{0}^{\pi}(\log C / t)^{\beta}|d \psi(t)|<\infty,
$$

then the series 


$$
\sum_{n=1}^{\infty} \frac{B_{n}(t)}{\{\log (n+1)\}^{1-\beta}} \text { is summable }\left|N, 1 /(n+1)\{\log (n+1)\}^{\alpha}\right|
$$

at $t=x$, where $0 \leqq \alpha<1, \beta \geqq 0$ and $\alpha+\beta<1$.

For $\alpha=\beta=0$, this corollary is due to R. D. Ram and N. Lal [15].

COROLlary 4. If

(i ) $\quad \int_{0}^{\pi} t^{-1}(\log C / t)^{-1}(\log \log C / t)^{\beta-1}|\psi(t)| d t<\infty \quad\left(\right.$ or $\left.\quad\left(\mathrm{i}^{\prime}\right) \psi(+0)=0\right)$

and

$$
\int_{0}^{\pi}(\log \log C / t)^{\beta}|d \psi(t)|<\infty,
$$

then the series

$$
\sum_{n=1}^{\infty} \frac{B_{n}(t)}{\log (n+1)\{\log \log (n+1)\}^{1-\beta}} \quad \text { is summable }|N, 1 /(n+1) \log (n+1)|
$$
at $t=x$, where $0 \leqq \beta<1$.

COROLlaRY 5. If

$$
\int_{0}^{\pi} t^{-1}|\psi(t)| d t<\infty \quad\left(\text { or } \quad\left(\mathrm{i}^{\prime}\right) \psi(+0)=0\right)
$$

and

$$
\int_{0}^{\pi}(\log C / t)|d \psi(t)|<\infty,
$$

then the series $\sum_{n=1}^{\infty} B_{n}(t)$ is summable $|N, \log (n+1) /(n+1)|$ at $t=x$.

CoROllary 6. If

$$
\int_{0}^{\pi} t^{-1}(\log C / t)^{-1}|\psi(t)| d t<\infty \quad\left(o r \quad\left(\mathrm{i}^{\prime}\right) \psi(+0)=0\right)
$$

and

$$
\int_{0}^{\pi}(\log \log C / t)|d \psi(t)|<\infty
$$

then the series

$$
\sum_{n=1}^{\infty} \frac{B_{n}(t)}{\log (n+1)} \quad \text { is summable }|N, \log \log (n+1) /(n+1) \log (n+1)|
$$

at $t=x$.

As these corollaries are similarly proved, we shall prove here only Corollary 6. 
Proof of CoRollary 6. In our theorems, we put

$$
p_{k}=\log \log (k+1) /(k+1) \log (k+1),
$$

$\lambda(t)=\log \log (t+1)$ and $\mu(t)=1 / \log (t+1)\{\log \log (t+1)\}$. Then we have

$$
P_{n}=\sum_{k=1}^{n} \log \log (k+1) /(k+1) \log (k+1) \simeq\{\log \log (n+1)\}^{2} .
$$

On the other hand,

$$
\sum_{k=n}^{\infty} \frac{\lambda(k) \mu(k)}{k P_{k}}=O\left(\frac{\log \log (n+1)}{\{\log \log (n+1)\}^{2}}\right)=O\left(\frac{\lambda(n)}{P_{n}}\right) .
$$

Therefore, by our theorems, we see that Corollary 6 holds.

Now, by Theorem 3, we see that the set of the conditions (i) and (ii) is equivalent to the set of the conditions ( $i^{\prime}$ ) and (ii) in the above corollaries except Corollary 2 where $\beta \neq 0$. However, we see from Lemmas 9 and 10 that the set of the conditions ( $\left.i^{\prime}\right)$ and (ii) is better than the set of the conditions (i) and (ii) in Corollary $2(\beta \neq 0)$.

For the Fourier analogue of these corollaries, the reader is referred to K. Kanno [8] and Y. Okuyama [13].

Finally, the author wishes to express his hearty thanks to Prof. K. Kanno for his valuable suggestions and encouragements in the preparation of this paper.

\section{REFERENCES}

[1] L. S. Bosanquet, The absolute Cesàro summability of a Fourier series, Proc. London Math. Soc., 41 (1936), 517-528.

[2] L. S. Bosanquet AND J. M. Hyslop, On the absolute summability of the allied series of a Fourier series, Math. Zeitschr., 42 (1937), 487-512.

[ 3 ] H. P. Dikshit, On a class of Nörlund means and Fourier series, Pacific J. Math., 33 (1970), 559-567.

[4] H. P. Dikshiт, Absolute Nörlund summability of a Fourier series with factors, preprint.

[5] H. P. Dikshit, On the absolute Nörlund summability factors of the conjugate series of a Fourier series, preprint.

[6] M. Izumi AND S. Izumi, Absolute Nörlund summability factor of Fourier series, Proc. Japan Acad., 46 (1970), 642-646.

[7] M. IzUmi AND S. IzUmi, Absolute Nörlund summability of Fourier series of function of bounded variation, Bull. Austral. Math. Soc., 3 (1970), 111-123.

[8] K. KANNo, On the absolute Nörlund summability of the factored Fourier series, Tôhoku Math. J., 21 (1969), 434-447.

[9] A. Kumar, Absolute summability theory and applications, D. Phil. Thesis, University of Jabalpur (1974).

[10] L. McFadden, Absolute Nörlund summability, Duke Math. J., 9 (1942), 168-207.

[11] R. Mohanty, The absolute Cesàro summability of some series associated with a Fourier series and its allied series, J. London Math. Soc., 25 (1950), 63-67.

[12] R. Mohanty, Absolute Cesàro summability of a series associated with a Fourier series, 
Bull. Calcutta Math. Soc., 44 (1952), 152-154.

[13] Y. OKuYama, On the absolute Nörlund summability factors of Fourier series, Bull. Austral. Math. Soc., 12 (1975), 9-21.

[14] T. PATI, On the absolute Nörlund summability of the conjugate series of a Fourier series, J. London Math. Soc., 38 (1963), 204-213.

[15] R. D. RAM AND N. LAL, On the behaviour of a series associated with the allied series of a Fourier series, Kōdai Math. Sem. Rep., 24 (1972), 131-135.

[16] T. SINGH, Absolute Nörlund summability of a factored Fourier series, Indian J. Math., 9 (1967), 227-236.

[17] O. P. VARShney, On the absolute harmonic summability of a series related to a Fourier series, Proc. Amer. Math. Soc., 10 (1959), 784-789.

[18] S. L. WANG, On the absolute Nörlund summability of Fourier series and its conjugate series, Acta Math. Sinica., 15 (1965), 559-573 (Chinese.)

Department of Mathematics

FACULTY OF ENGINEERING

SHINSHU UNIVERSITY

NAGANO, JAPAN 
